\title{
Laparoscopic radical distal pancreatosplenectomy with celiac axis excision following neoadjuvant chemotherapy for locally advanced pancreatic cancer
}

\author{
Yeon Su Kim', Ji Su Kim ${ }^{2,3}$, Sung Hyun Kim ${ }^{2,3}$, Ho Kyoung Hwang ${ }^{2,3}$, Woo Jung Lee ${ }^{2,3}$, Chang Moo Kang ${ }^{2,3}$ \\ 'Department of Surgery, Yonsei University College of Medicine, Seoul, Korea, \\ ${ }^{2}$ Division of Hepatobiliary and Pancreatic Surgery, Department of Surgery, Yonsei University College of Medicine, Seoul, Korea, \\ ${ }^{3}$ Pancreatobiliary Cancer Center, Yonsei Cancer Center, Severance Hospital, Seoul, Korea
}

\begin{abstract}
A recent successful prospective randomized control study comparing open distal pancreatectomy with laparoscopic distal pancreatectomy (LDP) has shown that LDP is a safe and effective surgical modality in treating left-sided pancreatic pathological conditions requiring surgical extirpation. With the accumulating surgical experiences and improved surgical techniques, we recently reported several cases of successful LDP in advanced pancreatic cancer following neoadjuvant chemotherapy. Herein, we report a case of LDP with celiac axis resection (LDP-CAR) in locally advanced pancreatic cancer (LAPC) following neoadjuvant chemotherapy. A 58-yearold female with LAPC was referred to our institution. Computed tomography (CT) findings revealed a 24-mm mass in the pancreatic body that showed celiac artery (CA), common hepatic artery abutment. There was no abutment with superior mesenteric artery, superior mesenteric vein, and portal vein. From these findings, Neoadjuvant chemotherapy (FORFIRINOX) was performed biweekly. After 8 cycles of chemotherapy, the tumor size was slightly decreased $(24 \mathrm{~mm}$ to $16 \mathrm{~mm})$, but still abutting to CA. After 14 cycles of chemotherapy, CT revealed the same tumor size $(16 \mathrm{~mm})$ still abutting to CA. LDP-CAR was performed. Intraoperative ultrasonography gastric perfusion and hepatic perfusion were confirmed using indocyanine green. The patient recovered without complications and was discharged from the hospital nine days after the surgery.
\end{abstract}

Key Words: Pancreatectomy; Neoadjuvant therapy; Laparoscopic surgery

\section{INTRODUCTION}

Pancreatic cancer (PC) is one of the most lethal malignant diseases in gastrointestinal tract. Among the PC treatment options, surgical resection is the only potentially curative option. Unfortunately, resectable PC cases are less than 20\%, at diagnosis and even most patients with resected PC experience

Received: June 30, 2021, Revised: September 26, 2021,

Accepted: September 27, 2021

Corresponding author: Chang Moo Kang

Division of Hepatobiliary and Pancreatic Surgery, Department of Surgery,

Yonsei University College of Medicine, Ludlow faculty building, 50-1 Yonsei-ro,

Seodaemun-gu, Seoul 03722, Korea

Tel: +82-2-2228-2100, Fax: +82-2-313-8289, E-mail: cmkang@yuhs.ac

ORCID: https://orcid.org/0000-0002-5382-4658

Copyright (C) The Korean Association of Hepato-Biliary-Pancreatic Surgery

This is an Open Access article distributed under the terms of the Creative Commons Attr$\mathrm{BY}$ NC bution Non-Commercial License (http://creativecommons.org/licenses/by-nc/4.0) which
permits unrestricted non-commercial use, distribution, and reproduction in any medium, provided the original permits unrestricted
work is properly cited. recurrence, commonly in the liver, lung, and peritoneum, leading to high cancer-related mortality. However, with the introduction of potent chemotherapeutic agents, advancement of surgical technique, perioperative management, and easy access to medical check-ups, clinical situations seem to face a new paradigm.

A recent successful prospective randomized control study (RCT) comparing open distal pancreatectomy (DP) with laparoscopic distal pancreatectomy (LDP) has shown that LDP is a safe and effective surgical modality in treating left-sided pancreatic pathological conditions requiring surgical extirpation [1]. Although controversies are surrounding the use of LDP in managing left-sided PC [2], LDP has increasingly been demonstrated as a potential option for treating resectable PC [3].

In our institution, LDP has only been applied in well-selected left-sided PC, that fulfill the Yonsei criteria [4]. With the accumulating surgical experience and improved surgical techniques, we recently reported several cases of successful LDP 
in more advanced PC following neoadjuvant chemotherapy [5]. Herein, we report a case of LDP with celiac axis resection (LDP-CAR) in locally advanced pancreatic cancer (LAPC) following neoadjuvant chemotherapy. The oncologic role of advanced LDP is also discussed based on the currently published literature.

\section{CASE}

\section{Case presentation}

A 58-year-old female first visited a local hospital with abdominal pain that had lasted for a month. Abdominal and pelvic computed tomography (CT) showed pancreatic body cancer, and she was referred to our institution for further evaluation and treatment. She was taking anticoagulants for hypertension and had dyslipidemia, and anxiety disorders. She had no specific family history. Laboratory data revealed a mild elevation of amylase (145 U/L, normal rage 30-115 U/ L). Tumor markers, including carcinoembryonic antigen (1.51 ng/mg), cancer antigen-19-9 (CA19-9; $21.1 \mathrm{U} / \mathrm{mL})$ were within normal limits on the first visit in our institution.

A CT pancreas dynamic findings revealed a $24-\mathrm{mm}$ mass in the pancreatic body without direct organ invasion and distant metastasis. But the celiac artery (CA), and common hepatic artery (CHA) showed abutment (Fig. 1A, 1C). There was no abutment with the superior mesenteric artery (SMA), superior mesenteric vein (SMV), and portal vein. Magnetic resonance cholangiopancreatography revealed a pancreas body mass of 2.4 $\mathrm{cm}$ with perivascular spread along the splenic artery (SA) and suspiciously to the lateral side of the CA. Pancreatic duct was not dilated or obstructed and there was no abuttment or encasement with SMA and SMV. Common bile duct was $0.9 \mathrm{~cm}$ (borderline dilation) and suspicious of chronic cholangiopathy. Endoscopic ultrasonography-guided fine needle aspiration cytology was performed, and pathological findings revealed a poorly differentiated pancreatic ductal adenocarcinoma (PDAC). Positron emission tomography-computed tomography findings did not show distant metastasis but showed mild uptake at the left para-aortic area, possibly metastasis.

Based on these findings, the patient was diagnosed with LAPC. FORFIRINOX was given as biweekly neoadjuvant chemotherapy. At first, a full dose of the standard regimen was proposed (130 mg of Oxaliplatin, $306 \mathrm{mg}$ of leucovorin, 275.4 $\mathrm{mg}$ of irinotecan, and a total of $2,446 \mathrm{mg}$ of fluorouracil). Before the second dose of FOLFIRINOX, she developed neutropenia, which required skipping fluorouracil bolus. After 6 cycles of chemotherapy, the tumor size was slightly decreased (24 to $16 \mathrm{~mm}$ ), but still abutting to CA. However, during the fourth additional chemotherapy cycle, although it was stable, the state of the tumor remained unchanged. And the patient's neurotoxic symptoms worsened, so it was difficult to continue chemotherapy. The $75 \%$ dose reduction was done from the tenth cycle of chemotherapy and after a total of 14 chemotherapy cycles, the patient was hospitalized for surgery. A biliary CT just before the surgery revealed the same tumor size $(16 \mathrm{~mm})$, still abutting to CA (Fig. 1B, 1D), and there was no significantly enlarged lymph node in the abdomen. Without embolization of CHA, LDP-CAR was attempted on March 17th, 2021.
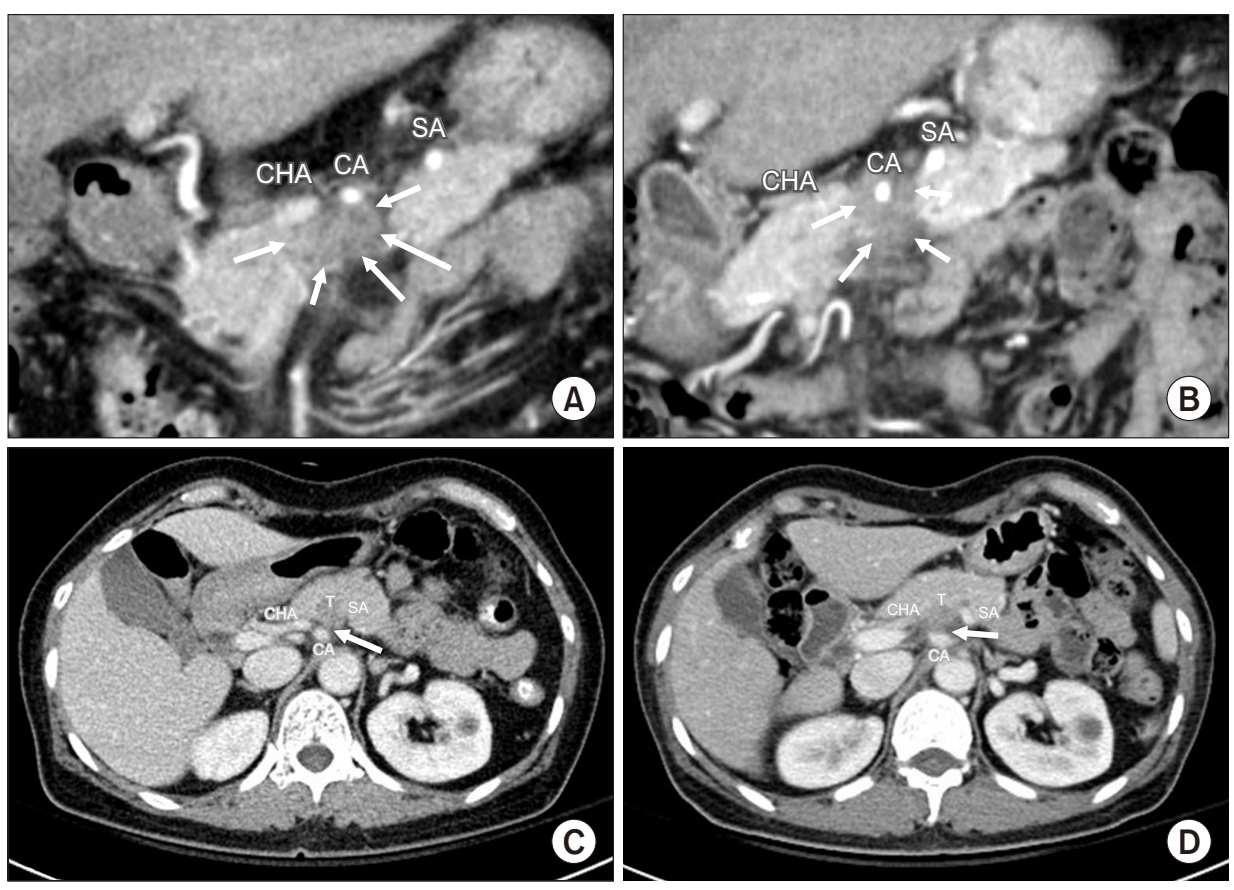

Fig. 1. Preoperative image. (A) Initial computed tomography (CT) scan. The tumor was abutting to the bifurcation site of $C A$ and CHA (coronal view). (B) Preoperative CT scan. The tumor was still abutting to $C A$ (coronal view). (C) Initial CT scan. The tumor was abutting to the bifurcation site of $C A$ and $\mathrm{CHA}$ (axial view). (D) Preoperative CT scan. The tumor was still abutting to CA (axial view). CA, celiac axis; CHA, common hepatic artery; $\mathrm{SA}$, splenic artery; $\mathrm{T}$, tumor. 

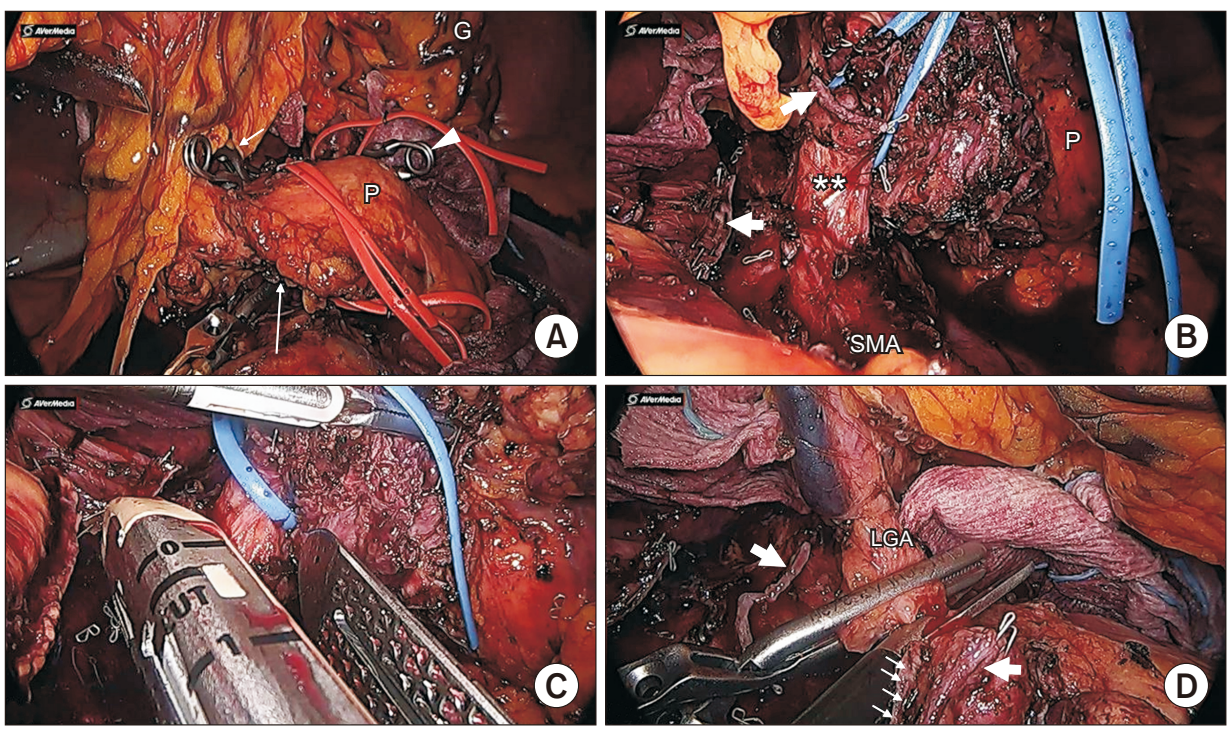

Fig. 2. Intraoperative finding. (A) Liver perfusion can be clearly evaluated after clamping common hepatic artery (CHA) (white short arrow), LGA (white arrowhead), and small collateral artery (white tall arrow). (B) Division of $\mathrm{CHA}$ (thick white arrows). Double asterisk indicates celiac artery (CA). (C) Division of CA. (D) Division of LGA, thick white arrows indicate resected $C A$. Thin white arrows indicate resected $\mathrm{CHA}$. G, stomach; P, pancreas; SMA, superior mesenteric artery; LGA, left gastric artery.

\section{Intraoperative findings}

Intraoperative findings showed neither peritoneal seeding nor distant metastasis. The tumor invaded the SA originating from the CA, left gastric artery (LGA), and CHA. There was no invasion around the SMA and SMV.

First, the possibility of preserving SMA was confirmed by separating the tumor bottom from the SMA. After successfully preserving the SMA, the LGA, CHA, and intrapancreatic collateral artery were all separately isolated. Because the distance between the tumor and the LGA was within $10 \mathrm{~mm}$, it was difficult to remove the tumor without cutting the LGA. Therefore, to confirm the possibility of combined CAR, we checked the liver and stomach perfusion after clamping all the LGA, CHA, and intrapancreatic collateral arteries (Fig. 2A). Intrahepatic artery pulsation was confirmed by intraoperative ultrasonogra- phy (IOUS) and there was no ischemic change in the stomach.

It was determined that resection seemed possible and the operation was performed. The neck of the pancreas was transected and the splenic vein was resected using a vascular stapler. For DP with splenectomy, all the ligaments around the pancreas and spleen were dissected. After checking where the SMA comes from the Aorta, the CA above it was dissected and isolated. Then, the LGA and CHA were resected and the CA was resected to the specimen side (Fig. 2B-2D). After the specimen was out (Fig. 3A), we confirmed gastric perfusion by indocyanine green (ICG) and hepatic perfusion by IOUS (Fig. 3B, 3C). The total operation time was 315 minutes, and blood loss was $350 \mathrm{~mL}$. No intraoperative transfusion was provided.
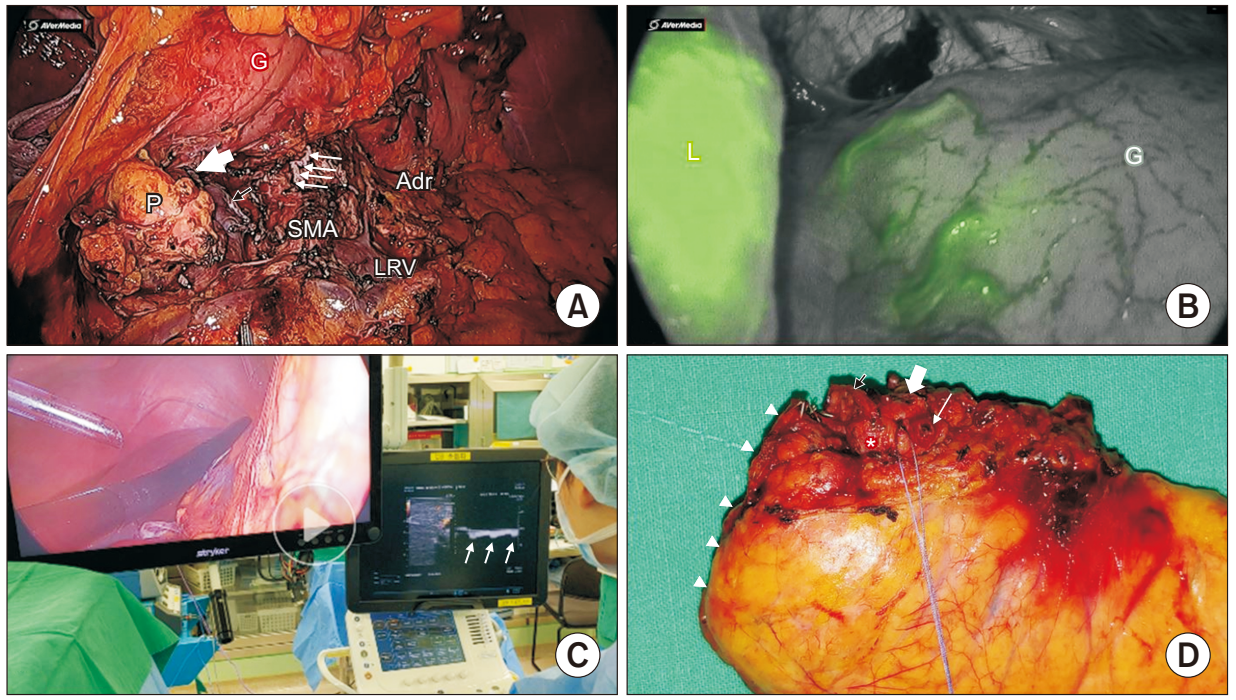

Fig. 3. Postoperative intraoperative ultrasonography (IOUS) and indocyanine green (ICG) gastric perfusion. (A) Postoperative view. The small multiple white arrows indicate resected celiac axis (CA). Black arrow means resected splenic vein. The large white arrow shows resected common hepatic artery (CHA). (B) ICG showed good perfusion of the remaining stomach. (C) IOUS demonstrated well-preserved intrahepatic pulsation (white arrows). (D) Surgical Specimen, white arrowheads indicate resection margin of the pancreas. Note the orifice resected CHA (black arrow), CA (thick white arrow), and left gastric artery (thin tall white arrow). $P$, pancreas; G, stomach; SMA, superior mesenteric artery; Adr, adrenal gland; LRV, left renal vein; $L$, liver, 


\section{Postoperative course}

The patient received postoperative care in the ward. Transfusion was not performed. She recovered routinely without complications. On the sixth postoperative day, there was no specific finding in the abdomen on routine CT. Histopathological findings showed $1.6 \times 1.4 \times 1.2 \mathrm{~cm}$ residual PDAC, and poorly differentiated and $1 / 10$ regional metastatic lymph node. The TNM classification (8th edition) was T1N1M0, stage IIb. All the resection margins including the CA was free from the tumor (Fig. 3D). After postoperative management, she recovered without complications and was discharged from the hospital nine days after surgery.

\section{DISCUSSION}

Since Appleby [6] reported multi-visceral resection for advanced gastric cancer, including en bloc gastrectomy, and distal pancreatosplenectomy with CAR, a modified version of this procedure has been applied in LAPC, as DP-CAR.

Based on single-center experiences, Professor Satoshi Hirano from Hokkaido University, Japan reported the largest series of DP-CAR for LAPC [7]. Eighty patients underwent DP-CAR for pancreatic body cancer and long-term survival outcomes were reported [7]. However, serious postoperative complications $(41.4 \%)$ including ischemic gastropathy $(28.8 \%)$, and delayed gastric emptying (25\%) were also reported [7].

Yamamoto et al. [8] investigated the effectiveness of DP-CAR in LAPC following neoadjuvant treatment. They analyzed 72 patients (18.3\%) with DP-CAR out of 395 patients with DP for PC. It found that the overall morbidity was significantly higher, but the median survival in patients with adjuvant chemotherapy demonstrated better survival outcomes than those in patients with R1-DP [8]. In addition, Klompmaker et al. [9] conducted a pan-European retrospective cohort study about the oncologic outcome of DP-CAR. They evaluated 69 patients with DP-CAR from 20 institutions in 12 countries and their results showed 53\% R0 resection, 25\% major morbidity, and $16 \%$ mortality with a comparable long-term survival outcome (median 18 months). These findings highlighted the importance of patients' selection and surgical expertise [10]. Gong et al. [11] recently conducted a meta-analysis on the safety and effectiveness of DP-CAR based on 291 patients from 18 literatures. It was reported that DP-CAR was associated with longer operation time, greater intraoperative bleeding, high incidence of venous (33.28\%), and arterial (11.53\%) reconstruction; however, there was comparable morbidity, mortality, and R0 rate with similar long-term survival [11]. Therefore, they concluded that DP-CAR was feasible and acceptable in terms of its survival benefits, but it should be performed with caution due to its potential postoperative morbidity [11]. In fact, in the 2020 French recommendation for PC with vascular involvement, pancreatectomy with combined arterial resection was proposed for clinical setting following neoadjuvant treatment [12].
In addition, the Korean clinical practice guideline for PC 2021 suggested that if radical resection is possible in patients with PC invading the CA, DP-CAR could be considered [13].

Following issues need to be considered; "Who will be a candidate for DP-CAR?" DP-CAR is likely to be applicable when PC invades the origin of the SA, where the margin-negative standard DP cannot be performed. Considering the realistic clinical situation, DP-CAR can be a potential option when determining resectability after neoadjuvant chemotherapy for LAPC [14]. According to our present experience, safe and effective DP-CAR may be difficult in cases of cancer overt invasion in the CA and at least 1 to $2 \mathrm{~cm}$ cancer-free space should exist for resection of CA from the aorta, which is the same reason suggested in the Yonsei criteria [4].

Is preoperative arterial embolization required? When the CA is excised, since the collateral from SMA is responsible for liver perfusion and the remaining stomach via an inferior pancreaticoduodenal artery, gastroduodenal artery, and proper hepatic artery, it is necessary to preserve and confirm this arcade during DP-CAR. It is still controversial whether preoperative embolization is necessary. In fact, there seem to be many centers that perform DP-CAR without preoperative embolization. In this case, we omitted preoperative arterial embolization, but confirmed preserved hepatic arterial flow before performing DP-CAR. After clamping all the three potential arteries (intrapancreatic collateral vessel, CHA, and even LGA), well-perfused hepatic arterial flow could be detected in IOUS.

Is it safe to perform an LGA resection? Conventional DPCAR includes the resection of LGA origin from CA. The resection of LGA will be determine by its location [15]. Modified DP-CAR is performed in patients with antecedent branching of LGA and the distance between the LGA and carcinoma is greater than $10 \mathrm{~mm}$ [15]. Modified DP-CAR is a method of excising CA while preserving LGA. In this case, the distance between the Tumor and LGA was close, to within $10 \mathrm{~mm}$, so conventional DP-CAR was performed.

A recent pooled analysis noted that ischemic gastropathy was $6 \%$, and combined total gastrectomy was required in $11 \%$ of the patients [16]. Ischemia of the stomach due to decreased gastric blood can result in gastric ulcer and consequently gastric bleeding [16]. Therefore, care should be taken in managing LGA. In case of combined resection of LGA like in this case, arterial reconstruction should be considered based on intraoperative ICG perfusion. A recent study suggested an intraoperative ICG-based reconstruction strategy, and further study should be performed regarding this issue [17].

Finally, is Laparoscopic DP-CAR safe? To the best knowledge of the authors, it was Cho et al. [18] who reported the first experiences of LDP-CAR for distal PC, but there were some patients' safety concerns. However, recently, there have been several laparoscopic and robotic DP-CAR studies $[18,19]$. In general, the question of which approach is better than the other is not important, the main focus should be a safe surgical 
technique that can achieve oncologic principles, and proper patients' selection that enables the patients' condition to be stable through chemotherapy, therefore, improving survival even after radically extended surgery [20].

In conclusion, according to the present case and the existing literature, LDP-CAR is feasible and safe. The role of DPCAR will be highlighted in an era of potent chemotherapeutic agents. However, it should be carefully considered based on patient selection and surgical experiences. Further study is mandatory.

\section{FUNDING}

None.

\section{CONFLICT OF INTEREST}

No potential conflict of interest relevant to this article was reported.

\section{ORCID}

Yeon Su Kim, https://orcid.org/0000-0003-4627-654X

Ji Su Kim, https://orcid.org/0000-0002-9501-9665

Sung Hyun Kim, https://orcid.org/0000-0001-7683-9687

Ho Kyoung Hwang, https://orcid.org/0000-0003-4064-7776

Woo Jung Lee, https://orcid.org/0000-0001-9273-261X

Chang Moo Kang, https://orcid.org/0000-0002-5382-4658

\section{AUTHOR CONTRIBUTIONS}

Conceptualization: YSK. Data curation: All authors. Methodology: YSK, JSK, CMK. Visualization: YSK, JSK. Writing - original draft: YSK, JSK, CMK. Writing - review \& editing: YSK, CMK.

\section{REFERENCES}

1. de Rooij T, van Hilst J, van Santvoort H, Boerma D, van den Boezem P, Daams F, et al. Minimally invasive versus open distal pancreatectomy (LEOPARD): a multicenter patient-blinded randomized controlled trial. Ann Surg 2019;269:2-9.

2. van Hilst J, de Graaf N, Abu Hilal M, Besselink MG. The landmark series: minimally invasive pancreatic resection. Ann Surg Oncol 2021;28:1447-1456.

3. Kantor O, Bryan DS, Talamonti MS, Lutfi W, Sharpe S, Winchester DJ, et al. Laparoscopic distal pancreatectomy for cancer provides oncologic outcomes and overall survival identical to open distal pancreatectomy. J Gastrointest Surg 2017;21:1620-1625.

4. Kang CM, Lee SH, Lee WJ. Minimally invasive radical pancreatectomy for left-sided pancreatic cancer: current status and future perspectives. World J Gastroenterol 2014;20:2343-2351.

5. Hong SS, Hwang HK, Lee WJ, Kang CM. Feasibility and safety of lap- aroscopic radical distal pancreatosplenectomy with adrenalectomy in advanced pancreatic cancer. Ann Surg Oncol 2020;27:5235-5236.

6. Appleby LH. The coeliac axis in the expansion of the operation for gastric carcinoma. Cancer 1953;6:704-707.

7. Nakamura T, Hirano S, Noji T, Asano T, Okamura K, Tsuchikawa T, et al. Distal pancreatectomy with en bloc celiac axis resection (modified Appleby procedure) for locally advanced pancreatic body cancer: a single-center review of 80 consecutive patients. Ann Surg Oncol 2016;23(Suppl 5):969-975.

8. Yamamoto T, Satoi S, Kawai M, Motoi F, Sho M, Uemura KI, et al. Is distal pancreatectomy with en-bloc celiac axis resection effective for patients with locally advanced pancreatic ductal adenocarcinoma? -Multicenter surgical group study. Pancreatology 2018;18:106-113.

9. Klompmaker S, van Hilst J, Gerritsen SL, Adham M, Teresa Albiol Quer M, Bassi C, et al. Outcomes after distal pancreatectomy with celiac axis resection for pancreatic cancer: a Pan-European retrospective cohort study. Ann Surg Oncol 2018;25:1440-1447.

10. Klompmaker S, Besselink MG. ASO author reflections: distal pancreatectomy with celiac axis resection for locally advanced pancreatic cancer-patient selection and surgical experience are key. Ann Surg Oncol 2018;25(Suppl 3):828-829.

11. Gong H, Ma R, Gong J, Cai C, Song Z, Xu B. Distal pancreatectomy with en bloc celiac axis resection for locally advanced pancreatic cancer: a systematic review and meta-analysis. Medicine (Baltimore) 2016;95:e3061.

12. Delpero JR, Sauvanet A. Vascular resection for pancreatic cancer: 2019 French recommendations based on a literature review from 2008 to 6-2019. Front Oncol 2020;10:40.

13. Korean Pancreatic Cancer Clinical Guidelines Development Committee. 2021 Korean pancreatic cancer clinical guidelines [Internet]. Goyang: National Cancer Center 2021 [cited 2021 Jan 5]. Available from: https://www.guideline.or.kr/guide/view.php?number=1115\&cate $=\mathrm{A}$.

14. Sperti C, Berselli M, Pedrazzoli S. Distal pancreatectomy for bodytail pancreatic cancer: is there a role for celiac axis resection? Pancreatology 2010;10:491-498.

15. Okada KI, Kawai M, Hirono S, Miyazawa M, Kitahata Y, Ueno M, et al. Ischemic gastropathy after distal pancreatectomy with en bloc celiac axis resection for pancreatic body cancer. Langenbecks Arch Surg 2018;403:561-571.

16. Lan J, Chen Y, Wang S, Zhou Y. Distal pancreatectomy with en bloc celiac axis resection for pancreatic cancer: a pooled analysis of 109 cases. Updates Surg 2020;72:709-715.

17. Oba A, Inoue Y, Sato T, Ono Y, Mise Y, Ito H, et al. Impact of indocyanine green-fluorescence imaging on distal pancreatectomy with celiac axis resection combined with reconstruction of the left gastric artery. HPB (Oxford) 2019;21:619-625.

18. Cho A, Yamamoto H, Kainuma O, Ota T, Park S, Ikeda A, et al. Pure laparoscopic distal pancreatectomy with en bloc celiac axis resection. J Laparoendosc Adv Surg Tech A 2011;21:957-959.

19. Thomaschewski M, Zimmermann M, Honselmann K, Müller-Debus CF, Jacob F, Wellner UF, et al. [Robot-assisted distal pancreatectomy with en bloc celiac axis resection (modified Appleby procedure) after 
neoadjuvant therapy]. Zentralbl Chir 2021. https://doi.org/10.1055/ a-1333-3667 [in press] German.

20. Kang CM. ASO author reflections: from concept to real clinical prac- tice of laparoscopic distal pancreatectomy for left-sided pancreatic cancer. Ann Surg Oncol 2020;27:5237-5238. 